\title{
Aspirin promotes oligodendrocyte precursor cell proliferation and differentiation after white matter lesion
}

\author{
Jing Chen ${ }^{1 \dagger}$, Shilun Zuo ${ }^{1,2 \dagger}{ }^{\dagger}$, Jing Wang ${ }^{1}$, Jian Huang ${ }^{1}$, Xiao Zhang ${ }^{1}$, Yang Liu ${ }^{1}$, Yunxia Zhang ${ }^{1}$, Jun Zhao $^{1}$, \\ Junliang Han ${ }^{1}$, Lize Xiong ${ }^{3}$, Ming Shi ${ }^{1}{ }^{*}$ and Zhirong Liu ${ }^{1 *}$
}

${ }^{\prime}$ Department of Neurology, Xijing Hospital, Fourth Military Medical University, Xi'an, China

${ }^{2}$ Department of Neurosurgery, Southwest Hospital, Third Military Medical University, Chongqing, China

${ }^{3}$ Department of Anesthesiology, Xijing Hospital, Fourth Military Medical University, Xi'an, China

\section{Edited by:}

Antonio Camins, University of

Barcelona, Spain

\section{Reviewed by:}

Sandra Orozco-Suárez, Mexican Institute of Social Security, Mexico

Marta Vázquez, Universidad de la

República, Uruguay

*Correspondence:

Ming Shi and Zhirong Liu,

Department of Neurology, Xijing

Hospital, Fourth Military Medical

University, 127 Changle-Xi Road,

Xi'an 710032, China

e-mail: biomidas@163.com;

liuzhir@fmmu.edu.cnlink

${ }^{\dagger}$ Jing Chen and Shilun Zuo have contributed equally to this work.
Cerebral white matter lesion (WML) is one of the main causes for cognitive impairment and is often caused by chronic cerebral hypoperfusion. A line of evidence has shown that aspirin has neuroprotective effects and produces some benefits in long-term outcome and survival for ischemic stroke patients. However, whether aspirin exerts a protective effect against WML is still largely unknown. Here, we showed that aspirin could promote oligodendrocyte precursor cell (OPC) proliferation and differentiation into oligodendrocytes after WML. Male Sprague-Dawley rats were subjected to permanent bilateral common carotid artery occlusion, a well-established model for WML. Four weeks later, Morris water maze test showed an impairment of learning and memory ability of rat while aspirin treatment improved behavioral performance. Low dose of aspirin $(25 \mathrm{mg} / \mathrm{kg}$ ) was found to elevate the number of OPCs while relatively high doses $(100-200 \mathrm{mg} / \mathrm{kg})$ increased that of oligodendrocytes, and ameliorated WML-induced the thinning of myelin, as revealed by the electron microscope. Similarly, our in vitro study also showed that relatively low and high doses of aspirin enhanced OPC proliferation and differentiation into oligodendrocytes, respectively. Furthermore, we revealed that aspirin enhanced extracellular signal-related kinase (ERK) but inhibited RhoA activities. In summary, we provided the first evidence that aspirin can promote oligodendrogenesis and oligodendrocyte myelination after WML, which may involve ERK and RhoA pathways.

Keywords: aspirin, oligodendrocytes, oligodendrocyte precursor cells, white matter lesion, extracellular signalrelated kinase, RhoA

\section{INTRODUCTION}

Cerebral white matter lesions (WML) are observed in aging and stroke and constitute the core pathology of Binswanger disease, a form of subcortical vascular dementia (Shibata et al., 2004). These WML are believed to be responsible for cognitive impairment and are caused by chronic cerebral hypoperfusion (Pantoni and Garcia, 1997). There is an evidence suggesting that the cerebral white matter is as vulnerable to ischemia as cerebral gray matter (Pantoni et al., 1996). Dewar et al. (1999) emphasized that total brain protection, in which not only gray matter but also white matter is protected, is important and necessary.

However, damage to white matter, which is composed of myelinated axons and oligodendrocytes, has been largely neglected. Multiple mechanisms were involved in WML. For example, maturation-dependent vulnerability in the oligodendrocyte lineage was found in a hypoxic-ischemic injury model (Back et al., 2002). Oligodendrocytes are best known as the myelin-forming cells in the central nervous system (CNS). Oligodendrocyte precursor cells (OPCs) are immature oligodendrocytes and can differentiate into myelin-forming cells under certain conditions ( $\mathrm{Fu}$ et al., 2005). In the adult brain, mature myelinating oligodendrocytes are continuously produced from local OPCs residing in the brain parenchyma (Gensert and Goldman, 1997; Fancy et al.,
2004) and from precursor cells located in the subventricular zone (Picard-Riera et al., 2002; Fancy et al., 2004; Menn et al., 2006). Given their high migratory potential and their ability to differentiate into myelin-forming cells, subventricular neural stem cells (NSCs) represent an important endogenous source of OPCs for preserving the oligodendrocyte population in the white matter and for the repair of demyelinating injuries (Gonzalez-Perez and Alvarez-Buylla, 2011). Therefore, it is proposed that any drug, which can increase the number of OPCs and/or oligodendrocytes, may be beneficial for the treatment of WML.

Aspirin has become a standard treatment for acute ischemic stroke since it produces some benefit in long-term outcome and survival if given within 14 days of stroke onset (Hankey et al., 2003). Depending on its dosage, aspirin has a wide spectrum of pharmacological activities and multiple sites of action, which may contribute to the neuroprotection (Berger et al., 2004). So far, numerous studies have shown the neuroprotective effects of aspirin (Lorenzo Fernandez, 2002; Castillo et al., 2003; Vartiainen et al., 2003; Berger et al., 2004; Asanuma et al., 2012). For example, aspirin was found to be protective against ischemia-induced neuronal damage in animal model (Castillo et al., 2003; Berger et al., 2004) and in patients (Castillo et al., 2003), and against dopamine quinone-induced neurotoxicity (Asanuma et al., 2012). 
Based on the evidence above, we proposed that aspirin might exert a protective action against WML. Thus, in this study, using a well-established WML model induced by chronic cerebral hypoperfusion, we examined the effects of different doses of aspirin on rat learning and memory ability and the changes in the expression of oligodendrocyte lineage markers, and further explored underlying mechanisms of aspirin.

\section{MATERIALS AND METHODS IN VIVO EXPERIMENTS \\ Animals and WML model}

Adult male Sprague-Dawley (SD) rats (from the Fourth Military Medical University Laboratory Animals Center, Xi'an, China), weighing 250-300 g, were kept under standard housing conditions at a temperature between 20 and $23^{\circ} \mathrm{C}$, with a 12-h light-dark cycle and a relative humidity of $50 \%$. Rats were divided into three groups randomly, the normal group, the control group, and the aspirin group. Rats in the control and aspirin groups were undertaken with WML model induced by permanent bilateral common carotid artery occlusion, as described previously (Farkas et al., 2007). During the operation, the rat rectal temperature was monitored and maintained at $37 \pm 0.5^{\circ} \mathrm{C}$. Immediately after cerebral ischemia, the rats in aspirin group were received different doses $(25,50,100$, and $200 \mathrm{mg} / \mathrm{kg}$, dissolved in $10 \%$ warm ethanol) of aspirin (SigmaAldrich, St. Louis, MO, USA) daily for 4 weeks intraperitoneally, and those in the control group were received the same volume of ethanol. All experimental procedures were reviewed and approved by the Animal Studies Committee of Fourth Military Medical University, Xi'an, China and animal study was carried out with the established institutional guidelines regarding animal use and care.

\section{Spatial learning and memory test}

Morris water maze behavioral test was used to assess animal spatial learning and memory abilities, as described previously (Morris, 1984). During the training trials, the platform location was fixed and submerged under opaque water. Rats in each group $(n=5)$ received four trials per day for four consecutive days. Each cycle consisted of $90 \mathrm{~s}$ trials with $10 \mathrm{~min}$ in inter-trial interval. The time to reach the platform was recorded every time. Once rats located the platform, they were allowed to remain on it for $30 \mathrm{~s}$. The rats that failed to find the platform within 90 s were manually placed on the platform for $30 \mathrm{~s}$ either. The rats that failed during four consecutive days were excluded from the analysis because of the possibility of a visual disturbance. For the assessment of spatial memory retention, a 60 -s probe trial was conducted at the end of training, in which the platform was removed from the pool.

\section{Anxiety-related behavior tests}

Open field test was performed in a square arena $(60 \mathrm{~cm} \times 60 \mathrm{~cm})$, with a white floor divided into 36 squares $(10 \mathrm{~cm} \times 10 \mathrm{~cm})$, enclosed by continuous, $25 \mathrm{~cm}$-high walls made of black plexiglass. The arena was lit by two red-light lamps $(2 \times 60 \mathrm{~W})$ placed over its center. In this test, the 20 squares adjacent to the wall represent a protected field, named "arena periphery," while the other 16 squares represent an exposed field, or "arena center." The animals were routinely tested during the first half of the dark phase of their light/dark cycle. The test was initiated by placing a single rat in the middle of the arena and allowing it to move freely for $15 \mathrm{~min}$. Rat behaviors were continuously monitored under a video camera, and the total amount of time each rat spent in the arena center was recorded.

Elevated plus test was performed, as described previously (Carola et al., 2002). The test was initiated by placing a rat on the central platform of the maze, facing one of the open arms, and allowing it to move freely. Each session lasted $5 \mathrm{~min}$ and rat behavior was monitored under a video camera placed above the apparatus. Open-arm visits, closed-arm visits, and the total visits of rat were recorded.

\section{BrdU pulsing}

For differentiation assays, the rats were received $\operatorname{BrdU}(100 \mathrm{mg} / \mathrm{kg}$, i.p. Sigma) once a day for 14 days after operation and then sacrificed at 4 weeks for the observation of newly differentiated oligodendrocytes. For proliferation assays, the rats were received three pulses of $\mathrm{BrdU}(100 \mathrm{mg} / \mathrm{kg})$ within $24 \mathrm{~h}$ before the animals were sacrificed for the observation of proliferating OPCs.

\section{Electron microscope}

For electron microscopy, the tissues from epon-embedded corpus callosum were trimmed and reoriented so that ultrathin cross sections could be cut and treated with uranyl acetate and lead citrate. Electron micrographs were analyzed using the analysis DocuSystem (Soft Imaging System). At least 800 fibers from each rat ( $n=3$ per treatment) were analyzed. According to a previous study (Coetzee et al., 1996), axon diameter and fiber diameter were measured (axon diameter was defined as the axon diameter excluding myelin, in contrast to fiber diameter, which was defined as axon diameter plus myelin) and $G$-ratios, which were defined as the diameter of the axon divided by fiber diameter, were calculated.

\section{IN VITRO EXPERIMENTS \\ Neural stem cell culture}

Primary NSCs were isolated from the cerebral cortex of embryonic day (E) 14.5 SD rats, as described previously (Chojnacki and Weiss, 2008). The cells grew as free-floating aggregates and were harvested and mechanically dissociated to produce single cell suspension for replating every 4-6 days. After a minimum of three passages, single cell suspensions were plated on poly-L-lysine-coated (Sigma) coverslips at a density of $5 \times 10^{4}$ cells/coverslip, and maintained in a differentiation medium [DMEM/F12 (Gibco/Invitrogen, Carlsbad, CA, USA), 2\% B27 (Gibco) and 1\% fetal bovine serum (FBS, HyClone, Logan, UT, USA)] with treatment of aspirin $(0.1,0.5,1$, 5 , and $10 \mu \mathrm{M}$ ) or same volume of ethanol for 4 or 7 days. After fixed in $4 \%$ paraformaldehyde for $30 \mathrm{~min}$, the cells were processed for immunostaining, as described below.

\section{OPC culture}

Oligodendrocyte precursor cell culture and purification were performed according to a previous study (Niu et al., 2012). For the observation of OPC proliferation, $5 \times 10^{4}$ cells were seeded on coverslips and grew in modified OPC growth-medium (mOGM) containing $15 \%$ of B-104CM, 1\% N2 (Gibco) supplement, and $5 \mu \mathrm{g} / \mathrm{ml}$ insulin (Sigma). For the observation of OPC differentiation, OPCs on coverslips were cultured in mOGM containing $1 \%$ 
FBS. After OPCs were cultured for 1 day (for proliferation assay) or 3 days (for differentiation assay) with the treatment of different concentrations of aspirin $(0.1,0.5,1.0,5.0$, and $10 \mu \mathrm{M})$ and same volume of ethanol, the cells were fixed in $4 \%$ paraformaldehyde for $30 \mathrm{~min}$ and subjected to immunocytochemistry (see below). For Western blot, the cells at a density of $2 \times 10^{7}$ were cultured in mOGM containing $1 \%$ FBS with $10 \mu \mathrm{M}$ aspirin for 3 days, and then were harvested for Western blotting assay (see below).

\section{RhoA activity detection}

Cultured OPCs at a density of $1 \times 10^{7}$ were maintained in mOGM containing $1 \%$ FBS, supplemented with $10 \mu \mathrm{M}$ aspirin, $10 \mu \mathrm{M}$ aspirin plus PD98059 [extracellular signal-related kinase (ERK) inhibitor], and the same volume of ethanol, respectively. Then, cell lysates of each group were collected and processed for Western blot as previously described (Li et al., 2013b). The activity of RhoA (Rho-GTP) was measured with the Rho Activation Assay Kit (Cytoskeleton, Inc., Denver, CO, USA), according to the protocols provided by the manufacture.

\section{IMMUNOFLUORESCENCE STAINING}

For brain tissues, the rats in different groups $(n=5)$ were sacrificed and brains were cut into $30 \mu \mathrm{m}$ coronal floating sections through the representative corpus callosum (from the bregma 1.20 to $-5.04 \mathrm{~mm}$ ) using a Leica CM1900 cryostat (Germany). The sections were first incubated in $2 \mathrm{M} \mathrm{HCl}$ at $37^{\circ} \mathrm{C}$ for $30 \mathrm{~min}$ and neutralized with $0.1 \mathrm{M}$ borate buffer ( $\mathrm{pH} 8.5$ ) for $10 \mathrm{~min}$. For double immunofluorescence staining, floating sections were incubated with rat anti-BrdU (1:300, Abcam, Cambridge, UK) and mouse anti-NG2 (1:200, Millipore, Billerica, MA, USA) or rabbit anti-proteolipid protein (PLP, 1:1000, Abcam) in PBS containing $1 \%$ BSA and $0.3 \%$ Triton-X100 at $4^{\circ} \mathrm{C}$ overnight and then incubated with anti-rat IgG conjugated with Cy3 (1:200, Vector, Burlingame, CA, USA) or biotinylated anti-mouse/rabbit IgG (1:300, Vector) for $2 \mathrm{~h}$ at room temperature. After washing, the sections were reacted with Cy2-conjugated streptavidin (1:400; Jackson ImmunoResearch, West Grove, PA, USA) for $2 \mathrm{~h}$ at room temperature and then observed under a FV-1000 confocal microscope.

For cultured cells, the cells on the coverslips were incubated with the following primary antibodies: mouse anti-A2B5 (1:200, BD Pharmingen, Heidelberg, Germany), mouse anti-CNPase (1:500, Abcam), rabbit anti-Ki67 (1:1000, Abcam), rabbit anti$\operatorname{MBP}(1: 100$, Abcam), and mouse anti-O4 (1:300, BD Pharmingen) in PBS containing $1 \%$ BSA and $0.3 \%$ Triton-X100 at $4^{\circ} \mathrm{C}$ overnight: after washing, the cells were incubated with anti-mouse/rabbit IgG conjugated with Cy2 or Cy3 (1:300; Vector) for $1 \mathrm{~h}$ at room temperature. The nuclei were stained with Hoechst 33342 (SigmaAldrich) for $5 \mathrm{~min}$. Immunostaining signals were observed under a Leica DMIRB microscope.

\section{WESTERN BLOT}

Corpus callosum tissues and cultured cells were used for Western blotting analysis, as detailedly described by our previous study (Li et al., 2013b). Total proteins were extracted using a compartment protein extraction kit (Millipore) according to the manufacturer's instructions. After electrophoresed on 10\% SDSpolyacrylamide gels, proteins in the gels were transferred onto
PVDF membranes (Millipore), which were incubated with following antibodies: anti-CNPase (1:1000, Abcam), anti-GAPDH (1:3000, Kangwei Biotechnology, Shanghai, China), anti-MBP (1:1000, Santa Cruz Biotechnology, Santa Cruz, CA, USA), and anti-PDGF $\alpha$ R (1:500, Abcam). After extensive washing, the membranes were incubated with horseradish peroxidase-conjugated secondary antibody (1:10000, Kangwei Biotechnology) for $1 \mathrm{~h}$ at room temperature and developed using an enhanced chemiluminescence western blotting detection kit (Kangwei Biotechnology). All band signals were quantified using Image $(\mathrm{NIH})$, and the data acquired were normalized to GAPDH expression and further normalized to the control.

\section{QUANTIFICATION AND STATISTICAL ANALYSIS}

For quantitation of $\mathrm{PLP}^{+}, \mathrm{NG}^{+}, \mathrm{BrdU}^{+} / \mathrm{PLP}^{+}$, and $\mathrm{BrdU}^{+} / \mathrm{NG}^{+}$ cells, the immunolabeled cells in the corpus callosum were counted under the microscope at $400 \times$ magnification. The middle part of corpus callosum bordered by the bilateral cingula was chosen for counting. At least three to five independent brains were included and six coronal sections (about $300 \mu \mathrm{m}$ apart) of each brain were used for counting or analysis. The results were expressed as the number of immunolabeled cells per square millimeter. For quantitation of cultured cells, positive cells were counted in a visual field (about $1.0 \mathrm{~cm}^{2}$ ) at $200 \times$ and the results were expressed as a percentage to the total cells indicated by Hoechst 33342 staining. At least three to five independent experiments were performed for each assay.

All data were presented as mean \pm SD and analyzed using SPSS 16.0 software. Differences between groups in behavior tests and Western blot were assessed using one-way ANOVA followed by Tukey's post hoc test. Differences between aspirin groups and the control in vitro were assessed using Student's $t$-test. A probability of $p<0.05$ was considered statistically significant.

\section{RESULTS ASPIRIN IMPROVES ANIMAL LEARNING AND MEMORY ABILITY AFTER WML}

To examine the effects of aspirin on learning and memory ability after WML in rats, Morris water maze was used to evaluate their behavior performance. Our results showed that the escape latencies in rats subjected to cerebral ischemia were significantly longer than those in normal rats (Figures 1A,B). Though rats treated with aspirin $(25,50,100$, and $200 \mathrm{mg} / \mathrm{kg})$ were not different from those treated with ethanol controls during the 1-3 days training (Figure 1A), rats in aspirin groups took shorter time to find the platform at 4 days after training (Figure 1B). No significant difference was observed among all doses of aspirin treatment group. It was noted that there were no significant differences among rats in the various groups in swimming speed throughout four training days (Figure 1C). Additionally, it was reported that cognitive impairment was often accompanied with depression (Richard et al., 2013). To exclude possible effects of aspirin on anxiety and depression in rats, we performed open field and elevated plus maze tests. Our results showed that either the time spent in the arena center in open field (Figure 1D) or the ratio of open-arm visits in elevated plus maze test (Figure 1E), was not significantly different between the aspirin and control groups. Taken together, our 

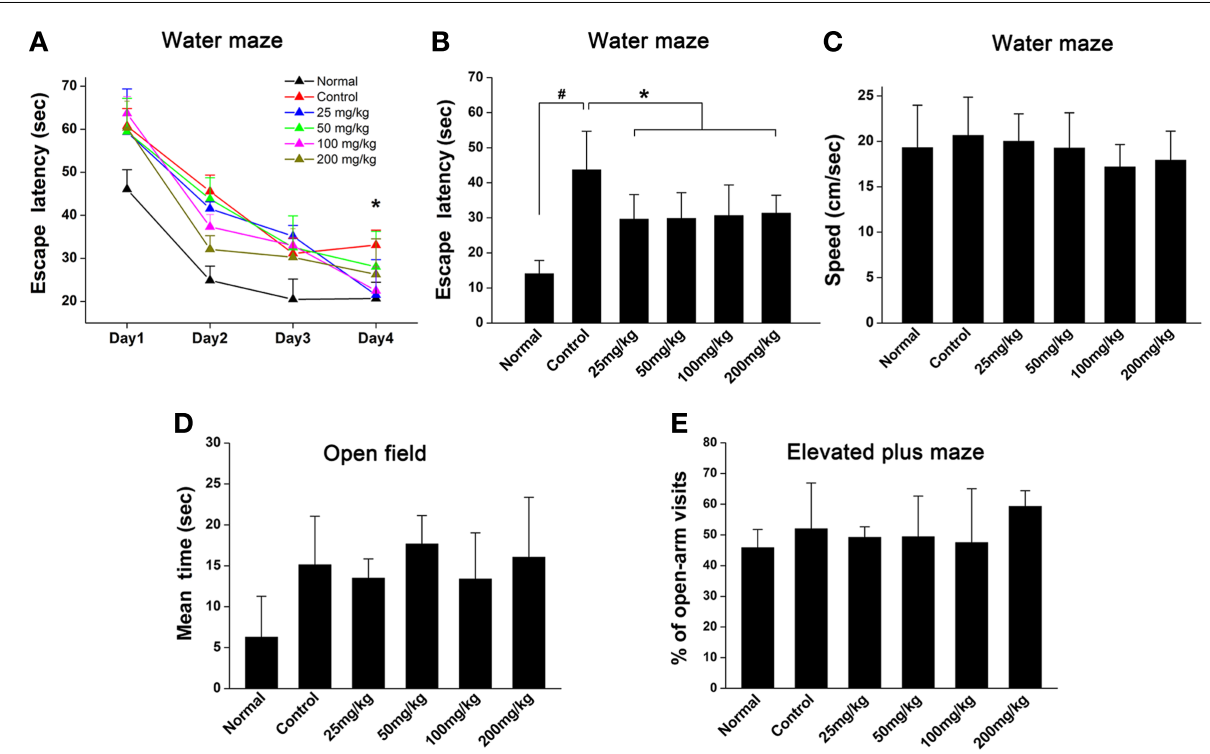

FIGURE 1 | Aspirin ameliorates cognitive impairment after WML. (A,B) Morris water maze was used for the evaluation of spatial learning in rats after cerebral ischemia. (A) Effects of different concentrations of aspirin on escape latency (time to find the hidden platform) plotted against training day. (B) Escape latency recorded at fourth training day. There was no significant difference among the aspirin groups, ${ }^{\#} p<0.05$ vs. the normal; ${ }^{*} p<0.05$ vs. the control. (C) Swimming speeds were not affected among different groups. (D,E) Analysis of animal anxiety-like behavior. Open field (C) and elevated plus maze (D) were used for the assessment of anxiety-like behavior. behavioral results indicate that aspirin can improve learning and memory ability after WML.

\section{ASPIRIN PROMOTES OPC PROLIFERATION AND DIFFERENTIATION AFTER WML}

To evaluate the effects of aspirin on oligodendroglia in the WML animal brain, we examined the changes of oligodendrocyte lineage in corpus callosum at 4 weeks after cerebral ischemia. Compared with the normal rats, the number of the cells expressing PLP, a marker for mature oligodendrocyte, was significantly decreased (Figures $\mathbf{2 A , C , G}$ ) in the control rats subjected to cerebral ischemia. However, aspirin treatment at doses of 25-200 mg/kg attenuated ischemia-induced reduction of oligodendrocytes (Figures 2C,E,G). Moreover, the number of NG2expressing OPCs were also increased after cerebral ischemia and further elevated with treatment of $25 \mathrm{mg} / \mathrm{kg}$ aspirin whereas 50,100 , and $200 \mathrm{mg} / \mathrm{kg}$ of aspirin did not have this effect (Figures 2B,D,F,H).

In order to investigate whether aspirin acts on the development of oligodendrocyte lineage, we examined OPC proliferation and differentiation by BrdU-pulse labeling assays. In the treatment of 100 and $25 \mathrm{mg} / \mathrm{kg}$ aspirin, the number of $\mathrm{PLP}^{+} / \mathrm{BrdU}^{+}$ double-stained new-differentiated oligodendrocytes and that of $\mathrm{NG}^{+} / \mathrm{BrdU}^{+}$double-labeled proliferating OPCs reached the peak (Figures 2A-F,I,J), respectively. In addition, we isolated the corpus callosum of each group and examined the protein levels of PDGF $\alpha \mathrm{R}$ (a marker for OPCs), CNPase (a marker for pre-oligodendrocytes), and MBP (a marker for mature oligodendrocytes) by Western blot. Immunoblotting results showed that the expression of PDGF $\alpha \mathrm{R}$ was upregulated after cerebral ischemia and further enhanced by $25 \mathrm{mg} / \mathrm{kg}$ aspirin treatment, which, however, was decreased by the treatment with 100 and $200 \mathrm{mg} / \mathrm{kg}$ aspirin (Figure 3A). By contrast, ischemia caused downregulation of MBP expression whereas $25-200 \mathrm{mg} / \mathrm{kg}$ aspirin ameliorated ischemia-induced decreased MBP expression. Noted that the MBP expression levels in $100 \mathrm{mg} / \mathrm{kg}$ aspirin-treated rats were higher than that in normal group (Figure 3B). Unexpectedly, aspirin did not affect CNPase expression (Figure 3C). Taken together, these results suggest that low dose of aspirin $(25 \mathrm{mg} / \mathrm{kg})$ may promote OPC proliferation while relatively high doses of aspirin $(100 \mathrm{mg} / \mathrm{kg})$ may enhance OPC differentiation into mature oligodendrocytes.

Furthermore, to investigate whether aspirin-induced increased mature oligodendrocytes can affect the myelin thickness after WML, we undertook an ultra-structural analysis of myelinated axons in the white matter tract of corpus callosum at 4 or 6 weeks after cerebral ischemia in rats with/without the treatment of $100 \mathrm{mg} / \mathrm{kg}$ aspirin. Compared with the normal, demyelination and reduction of myelin thickness were evident in the control rats subjected to ischemia (Figures 3D,E). Four-or six-week treatment of aspirin attenuated ischemia-induced demyelination and reduction of myelin thickness (Figures 3F,G). For quantitation, we assessed $G$-ratio, the ratio of axonal diameter to fiber diameter, which revealed an attenuated $G$-ratio in aspirin-treated rats, compared with the control (Figure $3 \mathbf{H}$ ), suggesting a recovery in the average myelin thickness with aspirin treatment. It was noted that the effect of 6-week treatment of aspirin was seemingly better than that of 4 -week treatment though with no significant difference (Figures $3 \mathbf{F}-\mathbf{H}$ ). Overall, our results demonstrate that depending on its dosage, aspirin may protect against ischemia-induced WML by affecting OPC fate and oligodendrocyte myelination. 

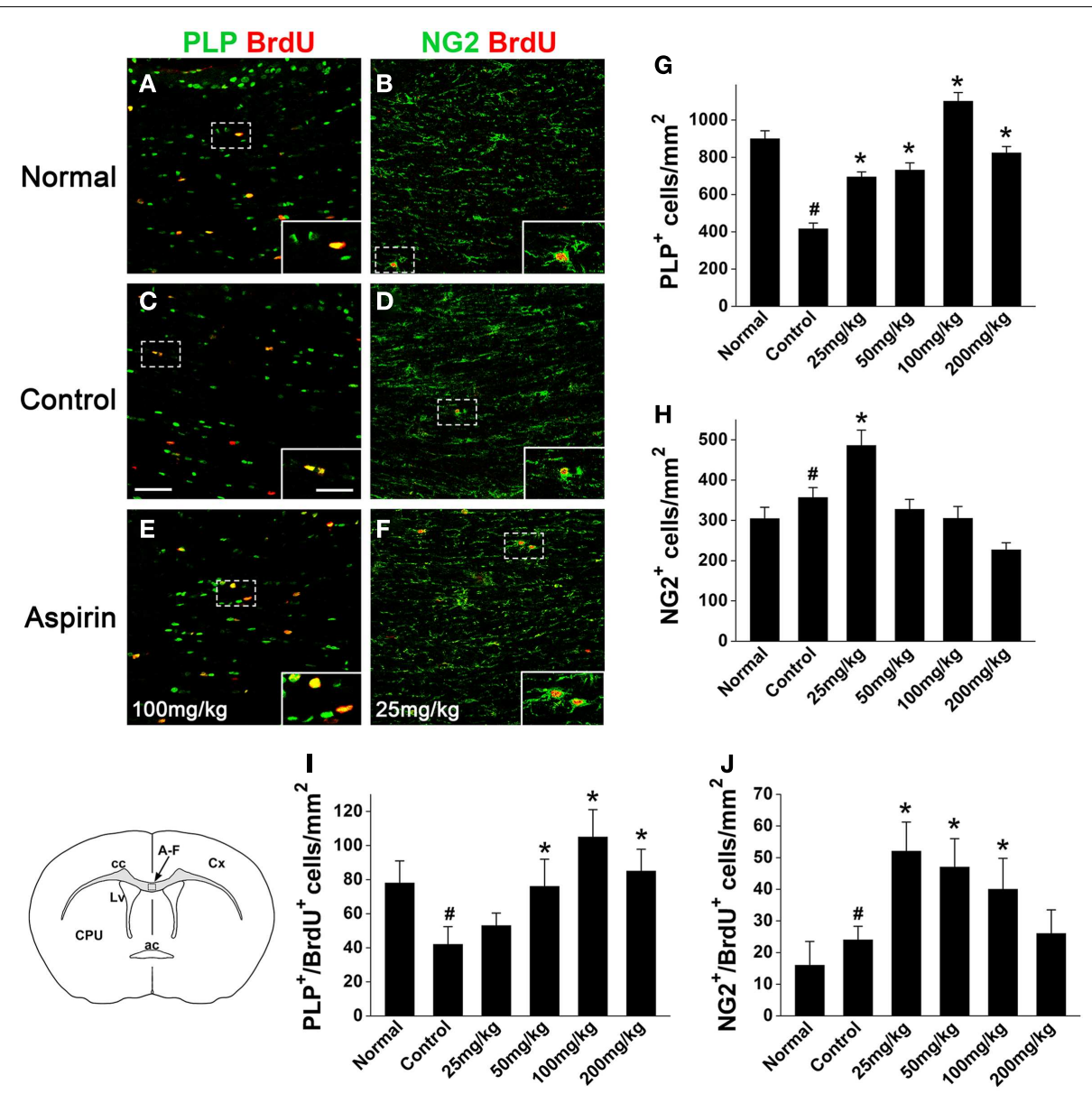

FIGURE 2 |Aspirin increases the numbers of oligodendrocytes and OPCs after WML. (A-F) Representative immunostaining micrographs showing oligodendrocytes and OPCs. Coronal sections across corpus callosum (as indicated in the left lower schematic) from the normal $(\mathbf{A}, \mathbf{B})$, control $(\mathbf{C}, \mathbf{D})$, and aspirin-treated $\mathbf{( E , F )}$ rats were double-stained with BrdU (red) and PLP [green; $(\mathbf{A}, \mathbf{C}, \mathbf{E})$ ] or NG2 [green; (B,D,F)]. Insets show higher magnification of the cells boxed in respective panels. Scale bars: $100 \mu \mathrm{m}$ (A-F), $25 \mu \mathrm{m}$ (insets). ac, Anterior commissure; cc, corpus callosum; Cx, cortex; CPU, caudate putamen; Lv, lateral ventricle. (G-J) Quantitation of the numbers of $\mathrm{PLP}^{+}(\mathbf{G}), \mathrm{NG}^{+} \mathbf{( H )}, \mathrm{PLP}^{+} / \mathrm{BrdU}^{+}$ (I), and $\mathrm{NG}^{+} / \mathrm{BrdU}^{+}$(J) cells in each group. Note that low dose of aspirin $(25 \mathrm{mg} / \mathrm{kg})$ increased NG2-expressing cells while relatively high doses of aspirin (100-200 mg/kg) increased PLP-expressing cells, ${ }^{*} p<0.05$ vs. the normal; ${ }^{*} p<0.05$ vs. the control.

\section{ASPIRIN PROMOTES OPC PROLIFERATION AND DIFFERENTIATION IN VITRO}

In order to confirm the effects of aspirin in vitro, we treated cultured OPCs with different concentrations of aspirin. Double staining of A2B5 (a marker for OPCs) and Ki67 (a marker for proliferating cells) was used for the observation of OPC proliferation. Our results showed that compared with the controls, more $\mathrm{A} 2 \mathrm{~B}^{+} / \mathrm{Ki} 67^{+}$cells were present with the treatment of $0.1 \mu \mathrm{M}$ aspirin whereas fewer were observed with the treatment of $10 \mu \mathrm{M}$ (Figures 4A-C,G). After OPCs were cultured in differentiation medium for 3 days, CNPase immunostaining was used for the evaluation of OPC differentiation. In the presence of $1.0-10 \mu \mathrm{M}$ aspirin, more $\mathrm{CNPase}^{+}$cells were observed than the controls while 0.1-0.5 $\mu \mathrm{M}$ did not have this effect (Figures 4D-F,H). Therefore, these in vitro data suggest that relatively low concentrations of aspirin could promote OPC proliferation while relatively high concentrations promote their differentiation.

\section{ASPIRIN PROMOTES NSC DIFFERENTIATION INTO OPCs AND OLIGODENDROCYTES IN VITRO}

Since NSCs are an important endogenous source of OPCs and sequential oligodendrocytes (Gonzalez-Perez and Alvarez-Buylla, 2011), we next investigated whether aspirin could affect the differentiation of NSCs into OPCs and oligodendrocytes. NSCs cultured in a differentiation medium for 4 and 7 days were immunostained with OPC marker O4 and oligodendrocyte markers CNPase or MBP, respectively. Compared with ethanol control, aspirin with different concentrations $(0.1-10 \mu \mathrm{M})$ increased the number of $\mathrm{O}^{+}$cells (Figures 5A,B,G). Differently, only relatively high concentrations of aspirin $(5.0-10 \mu \mathrm{M})$ increased the number of $\mathrm{CNPase}^{+}$or $\mathrm{MBP}^{+}$cells, as compared to the controls (Figures 5C-F,H,I). Importantly, we found that increased number of $\mathrm{CNPase}^{+}$or $\mathrm{MBP}^{+}$cells companied with decreased number of $\mathrm{GFAP}^{+}$cells (data not shown), indicating that the promotive effect of aspirin on NSCs differentiation into oligodendrocytes may be 

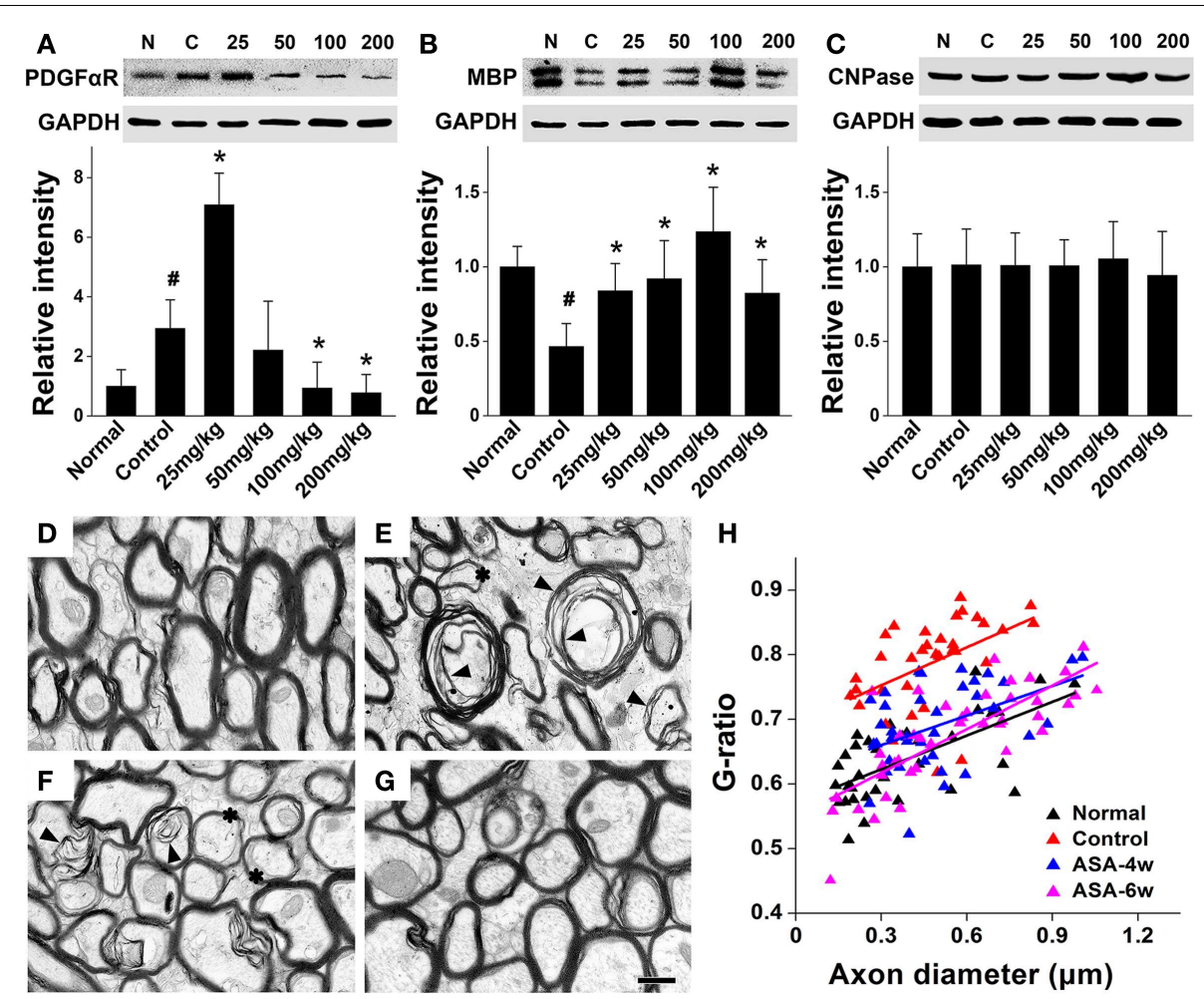

FIGURE 3 | Aspirin promotes the expression of oligodendrocyte proteins and myelin thickness after WML. (A-C) Western blotting analysis of OPC protein PDGF $\alpha$ R (A), myelin protein MBP (B), and pre-oligodendrocytes protein CNPase (C) from corpus callosum lysates of the normal, control, and aspirin-treated rats. Note that the maximum expression levels of PDGF $\alpha R$ and MBP were observed in the treatment of 25 and $100 \mathrm{mg} / \mathrm{kg}$ aspirin, respectively. GAPDH was used as an internal control, ${ }^{\sharp} p<0.05$ vs. the normal; * $p<0.05$ vs. the control. (D-G) Representative electron microscopy micrographs $(40,000 \times)$ of corpus callosum from the normal (D), control (E), $100 \mathrm{mg} / \mathrm{kg}$ aspirin-treated group at 4 weeks $(\mathbf{F})$, or 6 weeks

(G). Arrowheads indicate demyelination and asterisks show the thinning myelin. Scale bar: $0.5 \mu \mathrm{m}$. (H) Graphical representation of the $G$-ratio of individual fibers in relation to axon diameter (presented as scatter plots) at 6 weeks. Straight lines represent the linear regression line for each dataset. at the expense of astrocytes. These results imply that aspirin can promote the differentiation of NSCs into OPCs and sequential oligodendrocytes.

\section{ASPIRIN ENHANCES ERK AND INHIBITS RhOA ACTIVITIES}

A line of evidence has shown that p21Ras/MAPK (ERK) and Rho signaling pathways play crucial roles in oligodendroglial development (Stariha et al., 1997; Liang et al., 2004; YounesRapozo et al., 2009; Chew et al., 2010; Fyffe-Maricich et al., 2011; Zhao et al., 2013). Thus, to investigate the underlying mechanism(s) of aspirin, we first examined its effect on ERK1/2 pathway in OPCs cultured in a differentiation medium. Ten micromolar aspirin significantly upregulated MBP expression and enhanced ERK1/2 activity as revealed by increased phosphorERK expression, compared with ethanol control. However, addition of ERK inhibitor PD98059 (specific ERK kinase inhibitor; Tocris Cookson) either reversed the upregulated MBP expression and the enhanced ERK1/2 activity (Figures 6A,B). In addition, treatment of OPCs with aspirin reduced the activity of RhoA (approximate 80\%), compared with the control group (Figures 6C,D). Thus, these results indicate that aspirin may promote oligodendrogenesis via enhancing ERK and inhibiting RhoA activities.

\section{DISCUSSION}

In the present study, we provided the first evidence that aspirin could protect against WML-induced impairment of learning and memory ability and promote OPC proliferation and differentiation. This promotive effect of aspirin may involve its ability to enhance ERK and inhibit RhoA activities.

It is known that depression is always accompanied with cognitive impairment (Richard et al., 2013). In a previous study, some NSAIDS such as aspirin or drugs like simvastatin that have an antiinflammatory action could be useful in some depressive patients (Rahola, 2012). Furthermore, it was shown that aspirin could decrease the risk of depression in older men with high plasma homocysteine (Almeida et al., 2012). Thus, to confirm the beneficial effect of aspirin on impairment of learning and memory ability rather than depression after cerebral ischemia in the present study, we preformed open field and elevated plus maze test, and found that aspirin had no significant effects on anxiety and depression in rats.

Numerous clinical studies have shown that aspirin is widely used for stroke prevention. Depending on its dosage, aspirin has a wide spectrum of pharmacological activities and multiple sites of action. For example, its secondary preventive effects after ischemic events have been generally attributed to its antiplatelet actions 


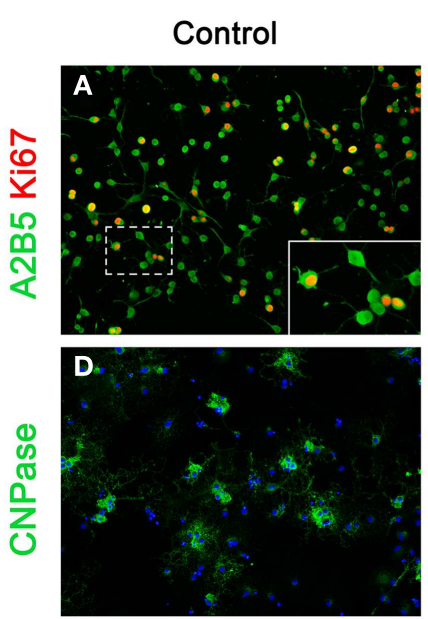

G

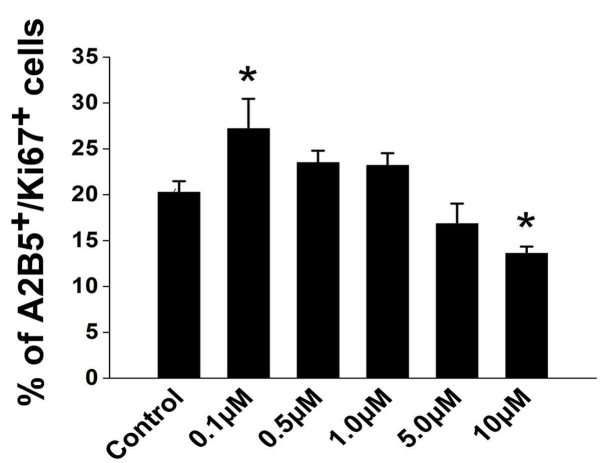

Aspirin $(0.1 \mu \mathrm{M})$
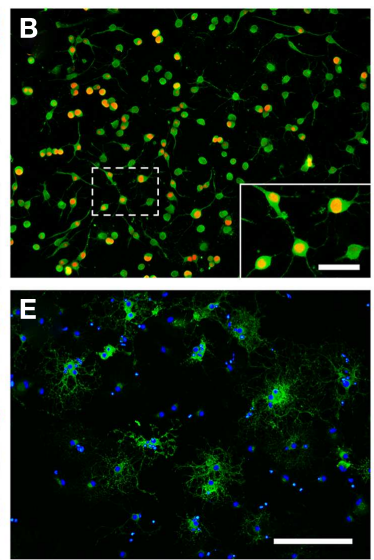

H

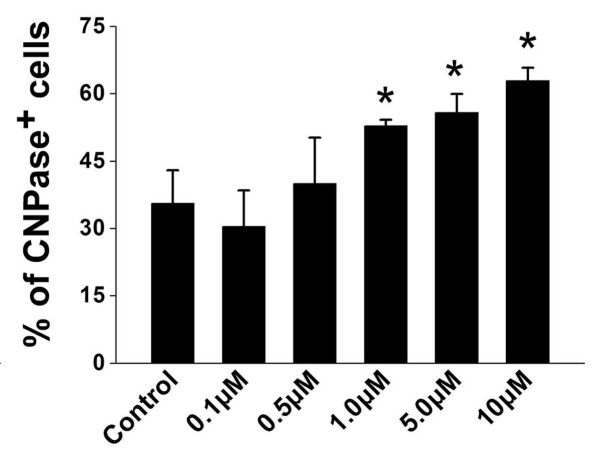

FIGURE 4 | Aspirin promotes OPC proliferation and differentiation in vitro. (A-F) Representative immunostaining micrographs showing OPC differentiation. Cultured OPCs treated with ethanol (control) or different concentrations of aspirin were double-stained with Ki67 (red) and A2B5 [green; (A-C)] or single-stained with CNPase [green; (D-F)].
Nuclei were counterstained with Hoechst 33342. Insets show higher magnification of the cells boxed in respective panels. Scale bars: $50 \mu \mathrm{m}$ (A-F), $25 \mu \mathrm{m}$ (insets). (G,H) Quantitation of the percentage of $\mathrm{Ki}^{+} 7^{+} / \mathrm{A} 2 \mathrm{B5}^{+}(\mathbf{G})$ and $\mathrm{CNPase}^{+}(\mathbf{H})$ cells to total cells in each group, ${ }^{*} p<0.05$ vs. the control. through the inhibition of cyclooxygenase, which is already reached at low doses (as low as 20-40 mg/day) (Group, 1991; van Gijn, 1992). Moreover, aspirin exerts antipyretic and analgesic effects at medium doses (about 2-4 g/day) and anti-inflammatory effects at high doses (6-8 g/day) in humans (Fitzgerald, 2004). It is widely acknowledged that in humans the metabolism of many drugs may vary considerably from rodents, and therefore must be adjusted for application in rodents. Therefore, according to an equation: dose (rat)/dose (human) $=\mathrm{BW}^{-0.25}$ (rat)/BW ${ }^{-0.25}$ (human) (BW = body weight), which scales human doses to rats (Hau, 2003), we chose $25 \mathrm{mg} / \mathrm{kg}$ (relatively low dose), 100-200 mg/kg (relatively high dose) for aspirin treatment in the present study.

Application of these doses of aspirin, interestingly we found in vivo and in vitro that aspirin can promote OPC proliferation at a relatively low dose while enhance their differentiation into oligodendrocytes and myelination at a relatively high dose (Figures 2-4). We observed that low dose of aspirin can upregulate the expression of OPC markers (e.g., NG2 and PDGF $\alpha$ R) but meantime suppress that of mature oligodendrocyte markers (e.g., PLP and MBP). By contrast, high dose of aspirin had just the opposite effects. We proposed that more OPCs were undergoing proliferation in the treatment of low dose rather that high dose of aspirin and vice versa. Additionally, we found that aspirin seemingly had little effect on the expression of pre-mature oligodendrocytes (CNPase), and this may reflect a balance state of oligodendrocyte lineage. In addition, cell loss or dysfunction has been shown to be responsible for animal behavior deficits. It was reported that hippocampal cell loss was possibly related to memory dysfunction (Smith et al., 1991). Loss of oligodendrocytes and of myelin was involved in vascular dementia patients after hypoxic-ischemic damage (Ihara et al., 2010), whereas oligodendrocyte remyelination was strongly correlated with the recovery of cognitive dysfunction following chronic cerebral ischemia (Chida et al., 2011). In the present study, aspirin could attenuated ischemia-induced demyelination and reduction of myelin thickness (Figure 2), probably accounting for the beneficial effects of aspirin on cognitive dysfunction.

In addition to local origin, OPCs are also arise from subventricular NSCs, an important endogenous source for preserving oligodendrocytes in the white matter and for the repair of 

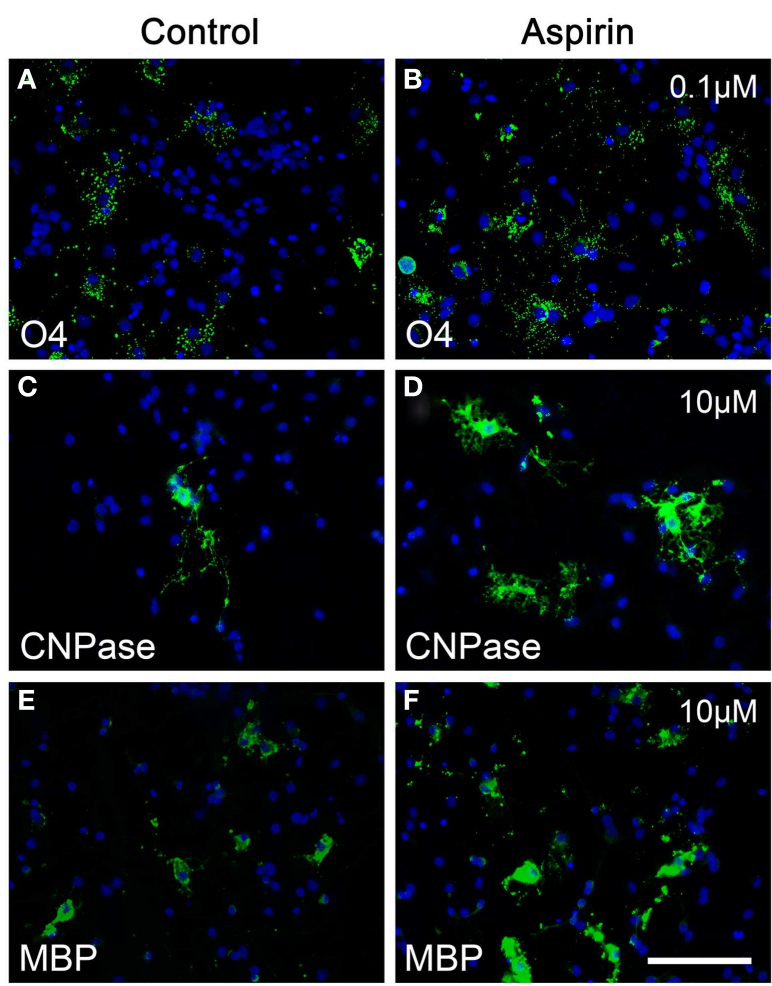
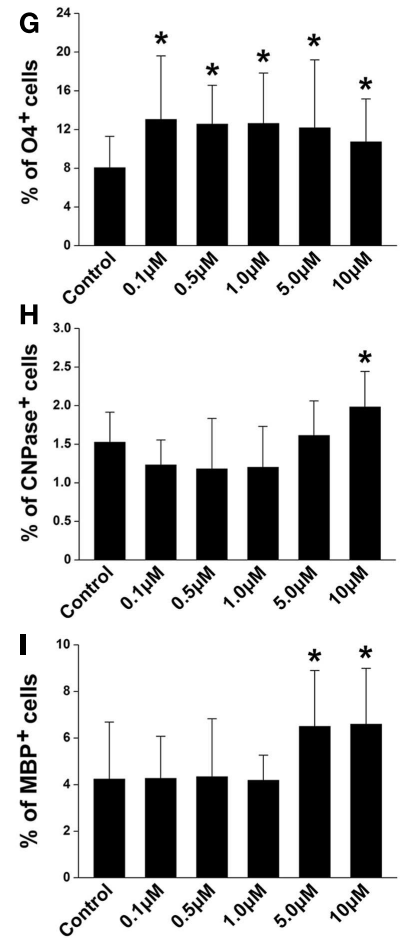

FIGURE 5 | Aspirin promotes NSC differentiation into OPCs and sequential oligodendrocytes. (A-F) Representative immunostaining micrographs showing NSC differentiation. Cultured NSCs treated with ethanol (control) or different concentrations of aspirin were immunostained with $\mathrm{O} 4$

(A,B), CNPase (C,D), or MBP (E,F). Nuclei were counterstained with Hoechst
33342. Scale bar: $50 \mu \mathrm{m}$. (G-I) Quantitation of the percentage of $\mathrm{O}^{+}$ (G), $\mathrm{CNPase}^{+} \mathbf{( H )}$, or $\mathrm{MBP}^{+}$(I) cells to all cells in each group. Note that although aspirin at different concentrations increased O4-expressing cells while only relatively high concentrations of aspirin $(5-10 \mu \mathrm{M})$ increased CNPase- or MBP-expressing cells, ${ }^{*} p<0.05$ vs. the control. demyelinating injuries. Although under normal conditions, the production of oligodendrocytes in the SVZ is scarce (Menn et al., 2006), demyelinating lesions in the neighboring white matter can significantly increase the generation of OPCs from SVZ progenitors (Nait-Oumesmar et al., 1999; Picard-Riera et al., 2002; Menn et al., 2006), which may also account for cerebral ischemia-induced upregulated expression of OPC markers (NG2 and PDGF $\alpha \mathrm{R})$ in the present study (Figures 2 and 3). Importantly, our in vitro data showed that aspirin could promote the differentiation of cultured NSCs into OPCs and sequential oligodendrocytes (Figure 5), indicating a possible beneficial function of aspirin in mobilizing endogenous NSCs against WML.

A group of studies have demonstrated that the effectiveness of aspirin in stroke treatment depends on various pathways beyond its antiplatelet and anti-inflammatory functions. Low dose aspirin was reported to inhibit the activity of RhoA (Li et al., 2013a), one of well-known members of the Rho GTPase family that regulates a wide range of fundamental cell functions such as proliferation and apoptosis. RhoA inactivation was found to enhance the proliferation of hematopoietic stem and progenitor cells (Ghiaur et al., 2006) and the differentiation and myelination in oligodendrocytes (Zhao et al., 2013). In addition, ERK pathways have particularly well-documented roles in proliferation and differentiation for various stem and precursor cells (Burdon et al., 2002). Constitutive activation of Erk1/2 signaling in oligodendrocytes not only caused myelination to occur earlier but also significantly induced more oligodendrocytes to myelinated in vitro (Xiao et al., 2012). Consistently, our in vitro results showed that aspirin could enhance ERK but inhibit RhoA activities (Figure 6), indicating aspirinmediated ERK activation and RhoA inhibition may be involved in OPC proliferation and differentiation. Intriguingly, a group of studies had shown that aspirin exerted an inhibitory effect on ERK activity in neuronal protection or apoptosis induction of carcinoma cells (Tegeder et al., 2001; Vartiainen et al., 2003; Park et al., 2010; Im and Jang, 2012). It is likely that this discrepancy of aspirin effectiveness may depend on different cell types and injury models used since these studies mainly focused on the effects of ERK on apoptosis during inflammation and tumorigenesis. By contrast, our study engaged in the effects of ERK on proliferation and differentiation during oligodendrocyte development.

In summary, we provide the first evidence that aspirin can promote OPC proliferation and differentiation and oligodendrocyte myelination after chronic cerebral ischemia by at least activating ERK and inhibiting RhoA activities. This promotive effect of aspirin on oligodendrocyte lineage may account for the recovery of ischemia-induced cognition impairment, suggesting a promising role of aspirin in the treatment of WML. 

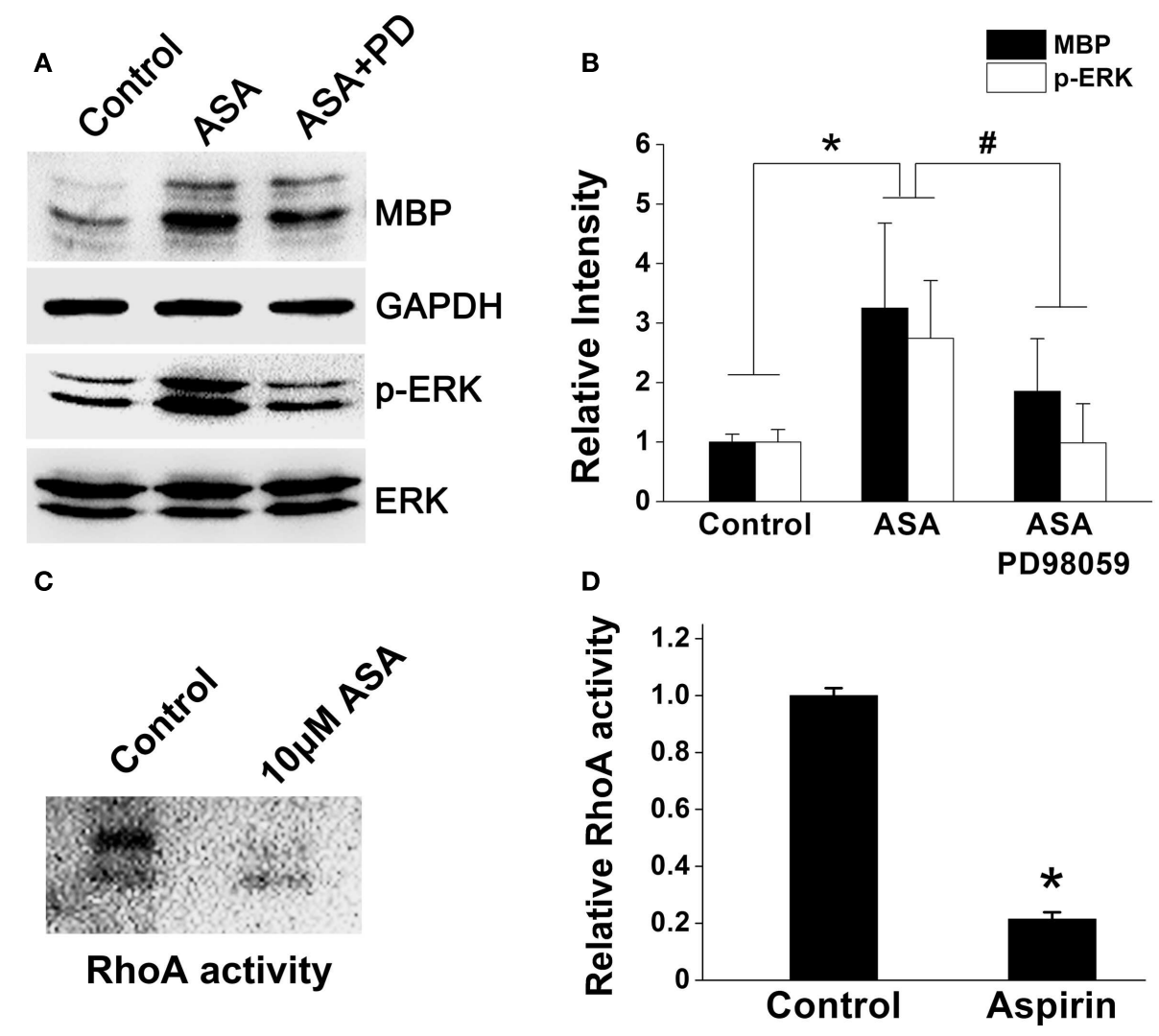

FIGURE 6 | Aspirin enhances ERK but inhibits RhoA activities. (A) Western blot analysis of MBP, phospho-ERK1/2, and total-ERK1/2 protein levels from cultured OPCs treated with ethanol, $10 \mu \mathrm{M}$ aspirin, or $10 \mu \mathrm{M}$ aspirin plus ERK1/2 inhibitor PD98059. Note that aspirin can enhance ERK activity as revealed by phospho-ERK1/2 protein level.

\section{ACKNOWLEDGMENTS}

The authors thank Ms. Dongyun Feng and Ms. Rui Wu for technical support. This study was supported by grants from the National Natural Science Foundation of China (No. 81070950 to Zhirong Liu and No. 31170801 to Ming Shi) and by Program for Changjiang Scholars and Innovative Research Team in University (No. IRT1053).

\section{REFERENCES}

Almeida, O. P., Flicker, L., Yeap, B. B., Alfonso, H., Mccaul, K., and Hankey, G. J. (2012). Aspirin decreases the risk of depression in older men with high plasma homocysteine. Transl. Psychiatry 2, e151. doi:10.1038/ tp.2012.79

Asanuma, M., Miyazaki, I., Kikkawa, Y., Kimoto, N., Takeshima, M., Murakami, S., et al. (2012). Cyclooxygenase-independent neuroprotective effects of aspirin against dopamine quinone-induced neurotoxicity. Neurochem. Res. 37, 1944-1951. doi:10.1007/s11064-012-0813-2

Back, S. A., Han, B. H., Luo, N. L., Chricton, C. A., Xanthoudakis, S., Tam, J., et al. (2002). Selective vulnerability of late oligodendrocyte progenitors to hypoxiaischemia. J. Neurosci. 22, 455-463.

Berger, C., Xia, F., Schabitz, W. R., Schwab, S., and Grau, A. (2004). High-dose aspirin is neuroprotective in a rat focal ischemia model. Brain Res. 998, 237-242. doi:10.1016/j.brainres.2003.11.049

Burdon, T., Smith, A., and Savatier, P. (2002). Signalling, cell cycle and pluripotency in embryonic stem cells. Trends Cell Biol. 12, 432-438. doi:10.1016/S09628924(02)02352-8

GAPDH was used as an internal control. (B) Quantitation of MBP, and phospho-ERK1/2 levels in each group, ${ }^{*} p<0.05$ vs. the control; ${ }^{\#} p<0.05$ vs. aspirin treatment group. (C,D) RhoA activity assay Aspirin inhibited RhoA activity by approximate $80 \%,{ }^{*} p<0.05$ vs. the control.

Carola, V., D’olimpio, F., Brunamonti, E., Mangia, F., and Renzi, P. (2002). Evaluation of the elevated plus-maze and open-field tests for the assessment of anxiety-related behaviour in inbred mice. Behav. Brain Res. 134, 49-57. doi:10.1016/S0166-4328(01)00452-1

Castillo, J., Leira, R., Moro, M. A., Lizasoain, I., Serena, J., and Davalos, A. (2003). Neuroprotective effects of aspirin in patients with acute cerebral infarction. $\mathrm{Neu}$ rosci. Lett. 339, 248-250. doi:10.1016/S0304-3940(03)00029-6

Chew, L. J., Coley, W., Cheng, Y., and Gallo, V. (2010). Mechanisms of regulation of oligodendrocyte development by p38 mitogen-activated protein kinase. J. Neurosci. 30, 11011-11027. doi:10.1523/JNEUROSCI.2546-10.2010

Chida, Y., Kokubo, Y., Sato, S., Kuge, A., Takemura, S., Kondo, R., et al. (2011). The alterations of oligodendrocyte, myelin in corpus callosum, and cognitive dysfunction following chronic cerebral ischemia in rats. Brain Res. 1414, 22-31. doi:10.1016/j.brainres.2011.07.026

Chojnacki, A., and Weiss, S. (2008). Production of neurons, astrocytes and oligodendrocytes from mammalian CNS stem cells. Nat. Protoc. 3, 935-940. doi:10. 1038/nprot.2008.55

Coetzee, T., Fujita, N., Dupree, J., Shi, R., Blight, A., Suzuki, K., et al. (1996). Myelination in the absence of galactocerebroside and sulfatide: normal structure with abnormal function and regional instability. Cell 86, 209-219. doi:10.1016/S00928674(00)80093-8

Dewar, D., Yam, P., and Mcculloch, J. (1999). Drug development for stroke: importance of protecting cerebral white matter. Eur. J. Pharmacol. 375, 41-50. doi:10.1016/S0014-2999(99)00280-0

Fancy, S. P., Zhao, C., and Franklin, R. J. (2004). Increased expression of Nkx2.2 and Olig2 identifies reactive oligodendrocyte progenitor cells responding to demyelination in the adult CNS. Mol. Cell. Neurosci. 27, 247-254. doi:10.1016/j.mcn. 2004.06.015 
Farkas, E., Luiten, P. G., and Bari, F. (2007). Permanent, bilateral common carotid artery occlusion in the rat: a model for chronic cerebral hypoperfusionrelated neurodegenerative diseases. Brain Res. Rev. 54, 162-180. doi:10.1016/j. brainresrev.2007.01.003

Fitzgerald, G. A. (2004). "Prostaglandins, aspirin and other NSAIDs," in Cecil Textbook of Medicine, eds L. Goldman, and D. Ausiello (Philadelphia: Saunders), 155-161.

Fu, S. L., Hu, J. G., Li, Y., Yin, L., Jin, J. Q., Xu, X. M., et al. (2005). Induction of rat neural stem cells into oligodendrocyte precursor cells. Sheng Li Xue Bao 57, 132-138. doi:10.3321/j.issn:0371-0874.2005.02.004

Fyffe-Maricich, S. L., Karlo, J. C., Landreth, G. E., and Miller, R. H. (2011). The ERK2 mitogen-activated protein kinase regulates the timing of oligodendrocyte differentiation. J. Neurosci. 31, 843-850. doi:10.1523/JNEUROSCI.3239-10.2011

Gensert, J. M., and Goldman, J. E. (1997). Endogenous progenitors remyelinate demyelinated axons in the adult CNS. Neuron 19, 197-203. doi:10.1016/S08966273(00)80359-1

Ghiaur, G., Lee, A., Bailey, J., Cancelas, J. A., Zheng, Y., and Williams, D. A. (2006). Inhibition of RhoA GTPase activity enhances hematopoietic stem and progenitor cell proliferation and engraftment. Blood 108, 2087-2094. doi:10.1182/ blood-2006-02-001560

Gonzalez-Perez, O., and Alvarez-Buylla, A. (2011). Oligodendrogenesis in the subventricular zone and the role of epidermal growth factor. Brain Res. Rev. 67, 147-156. doi:10.1016/j.brainresrev.2011.01.001

Group, T. D. T. T. S. (1991). A comparison of two doses of aspirin (30 mg vs. $283 \mathrm{mg}$ a day) in patients after a transient ischemic attack or minor ischemic stroke. The Dutch TIA Trial Study Group. N. Engl. J. Med. 325, 1261-1266. doi:10.1056/NEJM199110313251801

Hankey, G. J., Berge, E., and Sandercock, P. (2003). Anticoagulants versus antiplatelet agents for acute ischemic stroke. Stroke 34, 1571-1572. doi:10.1161/01.STR. 0000074551.42683 .98

Hau, J. (2003). "Animal models," in Handbook of Laboratory Animal Science, 2nd Edn, eds J. Hau, and G. L. Van Hoosier (Boca Raton: CRC Press), 8-15.

Ihara, M., Polvikoski, T. M., Hall, R., Slade, J. Y., Perry, R. H., Oakley, A. E., et al. (2010). Quantification of myelin loss in frontal lobe white matter in vascular dementia, Alzheimer's disease, and dementia with Lewy bodies. Acta Neuropathol. 119, 579-589. doi:10.1007/s00401-009-0635-8

Im, S. R., and Jang, Y. J. (2012). Aspirin enhances TRAIL-induced apoptosis via regulation of ERK1/2 activation in human cervical cancer cells. Biochem. Biophys. Res. Commun. 424, 65-70. doi:10.1016/j.bbrc.2012.06.067

Li, D. B., Yang, G. J., Xu, H. W., Fu, Z. X., Wang, S. W., and Hu, S. J. (2013a). Regulation on RhoA in vascular smooth muscle cells under inflammatory stimulation proposes a novel mechanism mediating the multiple-beneficial action of acetylsalicylic acid. Inflammation 36, 1403-1414. doi:10.1007/s10753-013-9680-5

Li, L., Liu, Z., Liu, J., Tai, X., Hu, X., Liu, X., et al. (2013b). Ginsenoside Rd attenuates beta-amyloid-induced tau phosphorylation by altering the functional balance of glycogen synthase kinase 3 beta and protein phosphatase 2A. Neurobiol. Dis. 54, 320-328. doi:10.1016/j.nbd.2013.01.002

Liang, X., Draghi, N. A., and Resh, M. D. (2004). Signaling from integrins to Fyn to Rho family GTPases regulates morphologic differentiation of oligodendrocytes. J. Neurosci. 24, 7140-7149. doi:10.1523/JNEUROSCI.5319-03.2004

Lorenzo Fernandez, P. (2002). Neuroprotection by aspirin in cerebrovascular pathology. An R Acad Nac Med (Madr) 119, 311-320; discussion 320-316.

Menn, B., Garcia-Verdugo, J. M., Yaschine, C., Gonzalez-Perez, O., Rowitch, D., and Alvarez-Buylla, A. (2006). Origin of oligodendrocytes in the subventricular zone of the adult brain. J. Neurosci. 26, 7907-7918. doi:10.1523/JNEUROSCI.129906.2006

Morris, R. (1984). Developments of a water-maze procedure for studying spatial learning in the rat. J. Neurosci. Methods 11, 47-60. doi:10.1016/0165-0270(84) 90007-4

Nait-Oumesmar, B., Decker, L., Lachapelle, F., Avellana-Adalid, V., Bachelin, C., and Baron-Van Evercooren, A. (1999). Progenitor cells of the adult mouse subventricular zone proliferate, migrate and differentiate into oligodendrocytes after demyelination. Eur. J. Neurosci. 11, 4357-4366. doi:10.1046/j.1460-9568.1999. 00873.x

Niu, J., Wang, L., Liu, S., Li, C., Kong, J., Shen, H. Y., et al. (2012). An efficient and economical culture approach for the enrichment of purified oligodendrocyte progenitor cells. J. Neurosci. Methods 209, 241-249. doi:10.1016/j.jneumeth. 2012.05.032
Pantoni, L., and Garcia, J. H. (1997). Pathogenesis of leukoaraiosis: a review. Stroke 28, 652-659. doi:10.1161/01.STR.28.3.652

Pantoni, L., Garcia, J. H., and Gutierrez, J. A. (1996). Cerebral white matter is highly vulnerable to ischemia. Stroke 27, 1641-1646. doi:10.1161/01.STR.27.9.1641

Park, I. S., Jo, J. R., Hong, H., Nam, K. Y., Kim, J. B., Hwang, S. H., et al. (2010). Aspirin induces apoptosis in YD-8 human oral squamous carcinoma cells through activation of caspases, down-regulation of Mcl-1, and inactivation of ERK-1/2 and AKT. Toxicol. In vitro 24, 713-720. doi:10.1016/j.tiv.2010.01.010

Picard-Riera, N., Decker, L., Delarasse, C., Goude, K., Nait-Oumesmar, B., Liblau, R., et al. (2002). Experimental autoimmune encephalomyelitis mobilizes neural progenitors from the subventricular zone to undergo oligodendrogenesis in adult mice. Proc. Natl. Acad. Sci. U.S.A. 99, 13211-13216. doi:10.1073/pnas.192314199

Rahola, J. G. (2012). Somatic drugs for psychiatric diseases: aspirin or simvastatin for depression? Curr. Neuropharmacol. 10, 139-158. doi:10.2174/ 157015912800604533

Richard, E., Reitz, C., Honig, L. H., Schupf, N., Tang, M. X., Manly, J. J., et al. (2013). Late-life depression, mild cognitive impairment, and dementia. JAMA Neurol. 70, 374-382. doi:10.1001/jamaneurol.2013.603

Shibata, M., Ohtani, R., Ihara, M., and Tomimoto, H. (2004). White matter lesions and glial activation in a novel mouse model of chronic cerebral hypoperfusion. Stroke 35, 2598-2603. doi:10.1161/01.STR.0000143725.19053.60

Smith, D. H., Okiyama, K., Thomas, M. J., Claussen, B., and Mcintosh, T. K. (1991) Evaluation of memory dysfunction following experimental brain injury using the Morris water maze. J. Neurotrauma 8, 259-269. doi:10.1089/neu.1991.8.259

Stariha, R. L., Kikuchi, S., Siow, Y. L., Pelech, S. L., Kim, M., and Kim, S. U. (1997) Role of extracellular signal-regulated protein kinases 1 and 2 in oligodendroglial process extension. J. Neurochem. 68, 945-953. doi:10.1046/j.1471-4159.1997. 68030945.x

Tegeder, I., Pfeilschifter, J., and Geisslinger, G. (2001). Cyclooxygenase-independent actions of cyclooxygenase inhibitors. FASEB J. 15, 2057-2072. doi:10.1096/fj.010390rev

van Gijn, J. (1992). Aspirin: dose and indications in modern stroke prevention. Neurol. Clin. 10, 193-207.discussion 208

Vartiainen, N., Goldsteins, G., Keksa-Goldsteine, V., Chan, P. H., and Koistinaho, J. (2003). Aspirin inhibits p44/42 mitogen-activated protein kinase and is protective against hypoxia/reoxygenation neuronal damage. Stroke 34, 752-757. doi:10.1161/01.STR.0000057813.31798.1F

Xiao, J., Ferner, A. H., Wong, A. W., Denham, M., Kilpatrick, T. J., and Murray, S. S. (2012). Extracellular signal-regulated kinase 1/2 signaling promotes oligodendrocyte myelination in vitro. J. Neurochem. 122, 1167-1180. doi:10.1111/j.14714159.2012.07871.x

Younes-Rapozo, V., Felgueiras, L. O., Viana, N. L., Fierro, I. M., Barja-Fidalgo, C., Manhaes, A. C., et al. (2009). A role for the MAPK/ERK pathway in oligodendroglial differentiation in vitro: stage specific effects on cell branching. Int. J. Dev. Neurosci. 27, 757-768. doi:10.1016/j.ijdevneu.2009.08.014

Zhao, C. F., Liu, Y., Que, H. P., Yang, S. G., Liu, T., Liu, Z. Q., et al. (2013). Rnh1 promotes differentiation and myelination via RhoA in oligodendrocytes. Cell Tissue Res. 353, 381-389. doi:10.1007/s00441-013-1625-7

Conflict of Interest Statement: The authors declare that the research was conducted in the absence of any commercial or financial relationships that could be construed as a potential conflict of interest.

Received: 02 December 2013; accepted: 12 January 2014; published online: 27 January 2014.

Citation: Chen J, Zuo S, Wang J, Huang J, Zhang X, Liu Y, Zhang Y, Zhao J, Han J, Xiong L, Shi M and Liu Z (2014) Aspirin promotes oligodendrocyte precursor cell proliferation and differentiation after white matter lesion. Front. Aging Neurosci. 6:7. doi: 10.3389/fnagi.2014.00007

This article was submitted to the journal Frontiers in Aging Neuroscience. Copyright ( 2014 Chen, Zuo, Wang, Huang, Zhang, Liu, Zhang, Zhao, Han, Xiong, Shi and Liu. This is an open-access article distributed under the terms of the Creative Commons Attribution License (CC BY). The use, distribution or reproduction in other forums is permitted, provided the original author(s) or licensor are credited and that the original publication in this journal is cited, in accordance with accepted academic practice. No use, distribution or reproduction is permitted which does not comply with these terms. 